\title{
An Investigation on Shape Morphing by Modulus Variation: Forward Approach
}

\author{
Amin Mohaghegh Motlagh and William W. Clark \\ Department of Mechanical Engineering, University of Pittsburgh, Pittsburgh, PA 15261, USA \\ Correspondence should be addressed to Amin Mohaghegh Motlagh; mohaghegh@gmail.com
}

Received 4 May 2014; Accepted 1 September 2014; Published 18 September 2014

Academic Editor: Parviz Malekzadeh

Copyright (c) 2014 A. Mohaghegh Motlagh and W. W. Clark. This is an open access article distributed under the Creative Commons Attribution License, which permits unrestricted use, distribution, and reproduction in any medium, provided the original work is properly cited.

\begin{abstract}
Structural shape deformation, in its conventional way, includes applying forces to a fixed-compliance structure to deform it to certain shapes. Rather than addressing shape control in the established way (applying forces to elastically or plastically deform a structure), this work studies the use of shape morphing, which involves combining applied forces and local modulus changes. Specifically in this paper, a simply supported elastic beam that can exhibit variable compliance behavior is selected as the model. This study focuses on the forward approach of morphing, that is, determining possible beam shapes due to the applied force and modulus variability. The goal is to incorporate variable-modulus materials into a structure model and utilize the controllable modulus change to quantify the morphing of the structure with limited actuator numbers, locations, and force levels. The resulting morphed shapes are quantified in terms of various characteristic parameters. The study demonstrates that a larger, and in some cases nonintuitive, space of shapes becomes possible when modulus change is utilized, for the same set of applied forces.
\end{abstract}

\section{Introduction}

The spatial orientation of a structure to one or several desired shapes may need to change in order to accommodate new geometry or stress requirements or to expand the performance capabilities and preserve energy metrics of the system. Traditional shape control methods have long been applied to a diverse range of structural materials, such as plastics, ceramics, metals, polymers, and composites whose mechanical properties are constant. For example, Reed et al. [1] developed a wing structure with capability of change in chord and wing's planform area, resulting in optimizing flight efficiency throughout the entire mission. Others used telescopic [2] and inflatable wings [3] for shape control. These methods accomplished a change in a structure's shape by continuously varying the magnitude, location, and number of actuation forces over the spatial domain to finally morph the system to the required geometry. Due to fixed material properties, the expected controlled shapes that arise from these methods are significantly limited to the actuators' capabilities as well as the material's range of stability. Lately, various adaptive materials have been extensively implemented in shape control applications. A group [4] developed a small-scale flying wing with active winglets via a distribution of piezo- or thermostrain actuators. Others [5] investigated the problem of determining the optimum piezoceramic actuator locations and voltages to minimize the error between the desired shape and the achieved shape in an Euler-Bernoulli beam model. Application of compact, lightweight, and robust shape memory alloy (SMA) actuators has been studied for morphing the shape of chevrons on the trailing edge of a jet engine in order to optimize acoustics and performance [6]. Following this work, the proposed SMA actuator system was developed by integrating the variable geometry chevrons (VGC) into the thrust reverser and performing the analysis and testing that was required to gain approval [7]. Chaudhry and Rogers [8] investigated shape control of beams in bending with induced SMA strain actuators. In each of these applications, a common thread is that the underlying structural material has fixed mechanical properties and the actuator is the sole contributor to the shape change. 
An alternative shape control method is to build the entire structure, or a significant portion, from an adaptive material whose compliance can be intelligently tuned based on the mechanical or spatial needs. The change of compliance in these systems may be localized or distributed. This change of shape due to modulus manipulation is referred to as shape morphing. Shape memory polymers (SMPs) have been utilized as a suitable structural material for morphing applications. SMP modulus-temperature relationship depends on the polymer type. For example, for SMP Veriflex, the modulus varies between $1050 \mathrm{MPa}$ in the glassy (i.e., hard) state and $0.24 \mathrm{MPa}$ in the rubbery (i.e., soft) state when the SMP is heated above its glass transition temperature, $T_{g}=62^{\circ} \mathrm{C}$ or $335.15 \mathrm{~K}$ [9]. Among many SMP applications, morphing aircraft has been more widely investigated. Experiments showed that SMP seems to be a promising component for a skin material of a morphing aircraft [10]. Later, Perkins et al. [11] fabricated a chord morphing wing using SMP. Rauscher [12] designed and fabricated a fiber-reinforced composite SMP morphing skin tile for a wing. As the wing spreads, each tile experiences a change in surface area that conventional passive material could hardly handle. Boyerinas et al. [13] developed a locking "smart" link mechanism for a folding wing by embedding SMA and SMP. In their design, the SMP layer served as the unlocking mechanism, whereas the SMA acted as an elastic support layer to provide structural integrity.

In order to achieve better morphing performance, SMP composites (SMPC) have been recently developed. These are shape memory polymers mixed into fillers such as spandex and carbon fibers which results in enhanced mechanical and flexibility properties of the material. Several works have investigated the utilization of SMPC in morphing applications. Chen et al. [14] studied the behaviors of SMP composite tubes in morphing skin applications. They developed a 3D model to study the modulus of multilaminated material and verified it using tensile test. Sun et al. [15] designed and manufactured a variable camber morphing wing with spandex fibers composite skins. The kinematic and kinetic simulations show the promising application for morphing wing structures. Leng et al. [16] presented a comprehensive work on the stimulus methods and applications of SMP on deployable and morphing structures.

Although significant work has been accomplished in structural shape control, including the use of shape memory materials, little attention has been given to the concept of explicitly combining modulus change with applied forces in order to enhance shape changing flexibility. The focus on this study is primarily to demonstrate the expansion of the spatial domain of a deformed structure due to modulus change. One of the most state-of-the-art smart materials are the Electroplastic elastomer hydrogels (EPEHs) that can reversibly switch between hard and soft states with the application of an electrical potential $[17,18]$. Unlike conventional intelligent materials, these materials do not exhibit any time response issue, heat-transfer induced phase transformation or heat energy dissipation. In addition, the focus of their response is only on modulus change, and not force application or shape change, so they are ideally suited for the type of morphing that is the focus of this work.
This paper explores concept of the shape morphing in a specific structure (i.e., a simply supported beam) with local modulus variation.

To elaborate on the primary motivation for the work, we will show that including modulus change in the structural shape change process enables not only increases in deformation, but also an expanded available space of shapes. For example, for the simply supported beam, if one wishes to deform a beam symmetrically, one can simply push on it in the middle; and to deform it nonsymmetrically, push it off to the side. But if one wants a nonsymmetrical deformation with actuation constrained to the middle, then there is no way to do it in a conventional, fixed-property beam (unless the boundary conditions are changed). Likewise, one is unlikely to create a symmetrically deformed shape with actuation constrained off-center. Transitioning the modulus at prescribed regions of the beam enables all of these possibilities to be done, without needing to move the force. This paper will illustrate this concept.

In general terms, morphing (or combining modulus change with applied forces) enables an expanded space of deformed shapes, referred to as morph space, over what can be achieved with conventional shape control with fixed modulus structures.

\section{Methods}

The goal is to apply various force combinations on the specific structure and explore what shapes can be obtained for (1) total beam in original hard modulus state, (2) total beam in soft modulus state, and (3) portions of the beam in hard or soft modulus states. The analysis in each stage involves applying governing elastic beam formulations while using the corresponding moduli to derive the analytical solution for the shape of the structure.

Figure 1 shows a simply supported beam whose modulus of elasticity can take on two different states at arbitrary locations along its length. Let $P$ be a transverse point load, applied at a distance $a$ from the left support, and let $L$ be the length of the beam.

For the sake of simplicity, distribution of moduli is described by a piecewise function. It is assumed that the moduli switch between two discrete values, that is, $E_{h}$, the hard (higher) modulus, and $E_{s}$, the soft (lower) modulus. The governing elastomechanical equations can now be defined by

$$
M(x)=E(x) I \frac{d^{2} y}{d x^{2}}=-\frac{P b}{L} x+P\langle x-a\rangle .
$$

This results in the following equation for beam deflection:

$$
y(x)=\frac{1}{E(x) I}\left(-\frac{P b}{6 L} x^{3}+\frac{1}{6} P\langle x-a\rangle^{3}+c_{1} x+c_{2}\right) .
$$

To fully determine the deflection (which defines the spatial shape) in (2), one must solve for the constants $c_{1}$ and $c_{2}$. Essential boundary conditions should be satisfied at the supports. For beams with different-modulus segments, additional deformation and slope continuity conditions at 


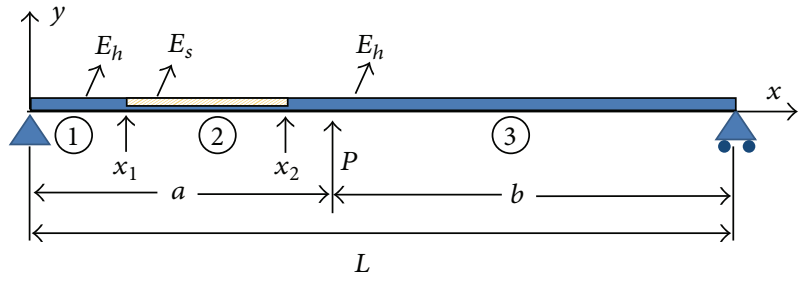

FIGURE 1: Loaded supported beam with variable moduli of elasticity.

the intermediate sections must also be fulfilled. For each segment, individual constants $c_{1}$ and $c_{2}$ are attributed. By applying these conditions, one gets the closed-form set of equations:

$$
\begin{aligned}
& {\left[\begin{array}{cccccc}
-E_{h}^{-1} & E_{s}^{-1} & 0 & 0 & 0 & 0 \\
0 & E_{s}^{-1} & -E_{h}^{-1} & 0 & 0 & 0 \\
0 & 0 & 0 & 1 & 0 & 0 \\
-x_{1} E_{h}^{-1} & x_{1} E_{s}^{-1} & 0 & -E_{h}^{-1} & E_{s}^{-1} & 0 \\
0 & x_{2} E_{s}^{-1} & -x_{2} E_{h}^{-1} & 0 & E_{s}^{-1} & -E_{h}^{-1} \\
0 & 0 & L & 0 & 0 & 1
\end{array}\right]\left[\begin{array}{c}
c_{1}^{1} \\
c_{1}^{2} \\
c_{1}^{3} \\
c_{2}^{1} \\
c_{2}^{2} \\
c_{2}^{3}
\end{array}\right]} \\
& =A\left\{\begin{array}{c}
f\left(x_{1}\right) \\
f\left(x_{2}\right) \\
0 \\
g\left(x_{1}\right) \\
g\left(x_{2}\right) \\
-A^{-1} g(L)
\end{array}\right\},
\end{aligned}
$$

where $c_{1}^{i}$ and $c_{2}^{i}$ are the coefficients corresponding to the $i$ th segment and $f(x), g(x)$, and $A$ are, respectively, defined as

$$
\begin{aligned}
f(x) & =-\frac{P b}{2 L} x^{2}+\frac{1}{2} P\langle x-a\rangle^{2} \\
g(x) & =-\frac{P b}{6 L} x^{3}+\frac{1}{6} P\langle x-a\rangle^{3} \\
A & =E_{h}^{-1}-E_{s}^{-1} .
\end{aligned}
$$

Solving for the coefficients, one may obtain the deformation of the beam by summation of piecewise displacement functions:

$$
y(x)=H_{1} y_{1}(x)+H_{2} y_{2}(x)+H_{3} y_{3}(x),
$$

where $y_{i}(x)$ is the deformation function of the $i$ th section and $H_{i}$ is the Heaviside function being unity only within the $i$ th section.

The general process to analyze the beam deflections is carried out as follows: a single transverse force set to be the maximum permissible force was constantly applied at the midspan of the hard beam and the deformation of the beam was calculated. Then, a section with certain length was softened and morphing of the beam under the same forcing condition was evaluated and plotted. This procedure was consecutively repeated for soft segments with various lengths. In order to maintain the material stability of the deflected structure for any arbitrary soft segment configuration, the force limit was set to the permissible elastic force in

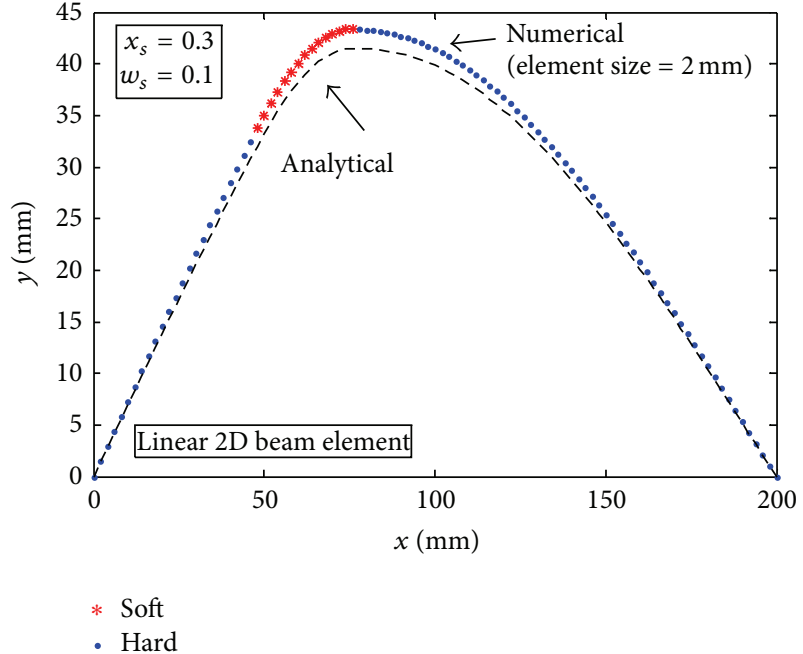

FIGURE 2: Analytical versus numerical displacement (coarse mesh).

the most compliant state of the beam (i.e., $P_{\max }=P_{Y}^{s}$ ). Using the elasticity equations relating stress, strain, and moment [19], the allowable force can be determined as follows:

$$
P_{Y}^{s}=0.002 E_{s}\left(\frac{I L}{a b c}\right)
$$

where $I$ is the second moment of section area and $L, a, b$, and $c$ are geometrical parameters representing beam length, distance from the point force to the supports, and half the cross section's height, respectively. In other words, the maximum allowable force is set such that the bending stress induced by the force does not exceed the smallest yield stress, which in the assumed linear elastic material states is proportional to the lowest modulus of the structure.

\section{Numerical Validation}

In order to verify the analytical results, a finite element study was performed. We developed routines to establish interaction between Abaqus Ver. 6.8.1 (Simulia Corp.) and MATLAB $^{\odot}$ R2010 (Mathworks Inc.) to simulate the shape deformation of the structure. A numerical 2D beam element with rectangular cross section $(20 \times 2 \mathrm{~mm})$ was chosen as the basic modeling element. An initial mesh size of $2 \mathrm{~mm}$ was assigned. To validate the numerical model, it was compared to the analytical solution, as derived above. Table 1 presents the specifications of the model.

Figure 2 shows example results of the comparison of deflected shapes with load at midspan. The analytical and numerical solutions are shown in which the beam contains a small softened segment defined by segment center point $x_{s}$ and length $w_{s}$. The soft segment is identified in the numerical solution with red cross symbols.

An approximate maximum error of $4 \%$ is observed in the vicinity of the peak point. To speculate on the source of error, finer elements with size decreasing by factors of 2 (i.e., element sizes of $1,0.5,0.25$, and $0.12 \mathrm{~mm}$ ) were replaced and the shape morphing was resimulated. The RMS error 


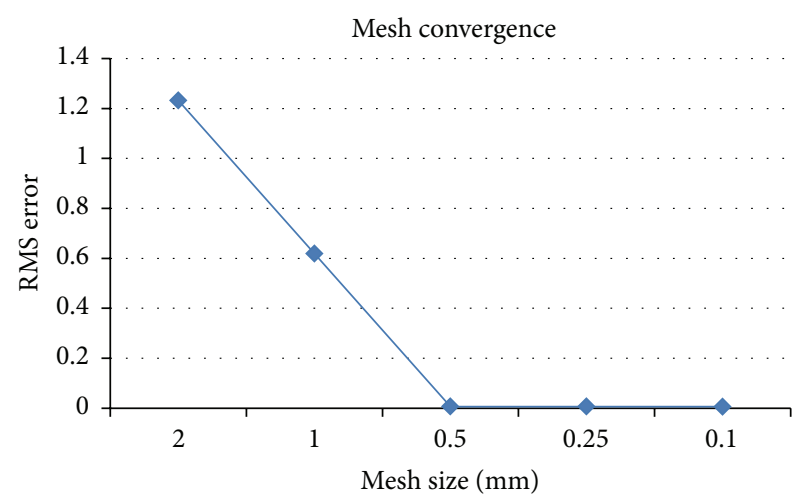

FIgURE 3: Mesh convergence for the numerical simulation.

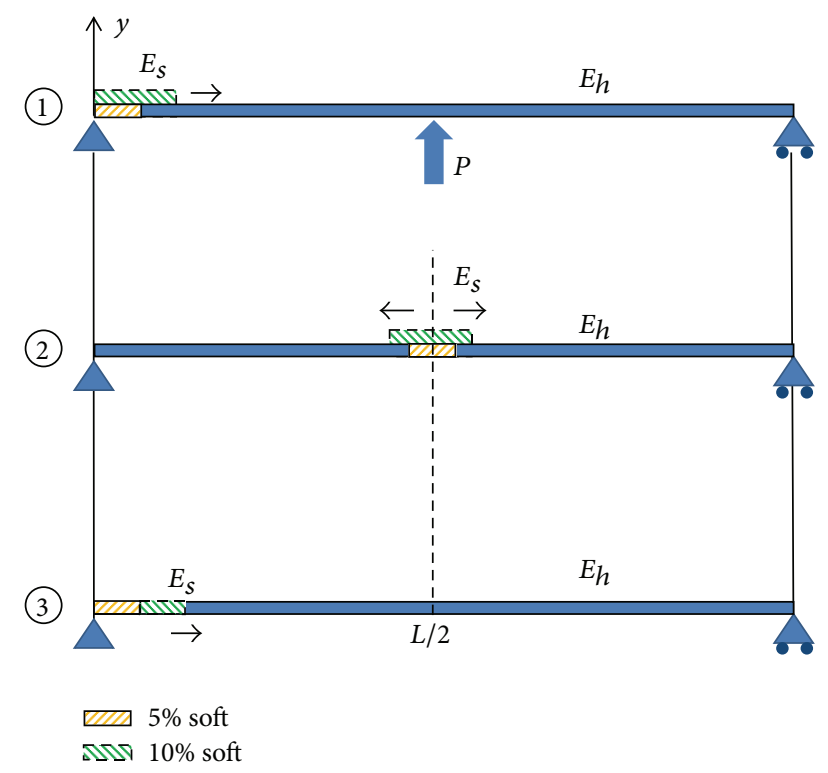

FIgURE 4: Three different patterns used in unisection morphing scheme: Case (1) transitioned section grows from the left (or right) end of the beam, Case (2) transitioned section grows symmetrically from the midspan, and Case (3) soft segment with a fixed length shifts through the structure from left end to right end.

was calculated at each simulation stage and the results were plotted in Figure 3. The FE solution has converged after $0.5 \mathrm{~mm}$ mesh. Therefore, this element size was used for further simulations.

\section{Results and Discussion}

To explore the various achievable shapes when the beam modulus locally changes, two primary morphing schemes were considered, unisection and bisection morphing. Each morphing scheme comprises certain arrangement of soft segments, as explained below.

(a) Unisection Morph Scheme. It is defined as only one section of the structure is softened. Figure 4 depicts three proposed morphing patterns in a unisection scheme. Soft segments

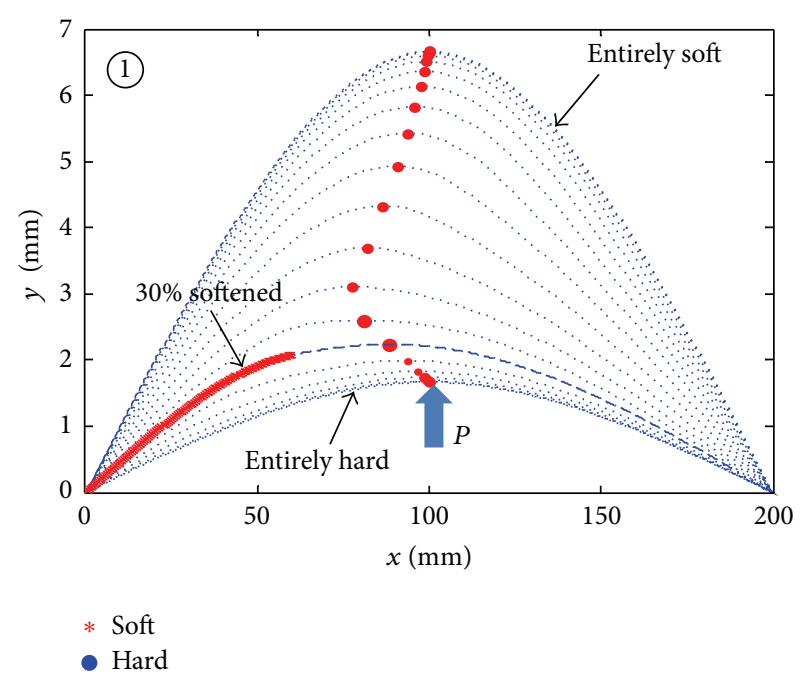

FIGURE 5: Morphed shapes with highlighted peak points (fixed soft segment at the left grows to the right with $5 \%$ size increments. Mirror peak locus will form by moving the sections from right to left). An example curve with $50 \%$ soft segment is highlighted. An envelope is formed by the loci of morphed shapes' peaks and their mirror image.

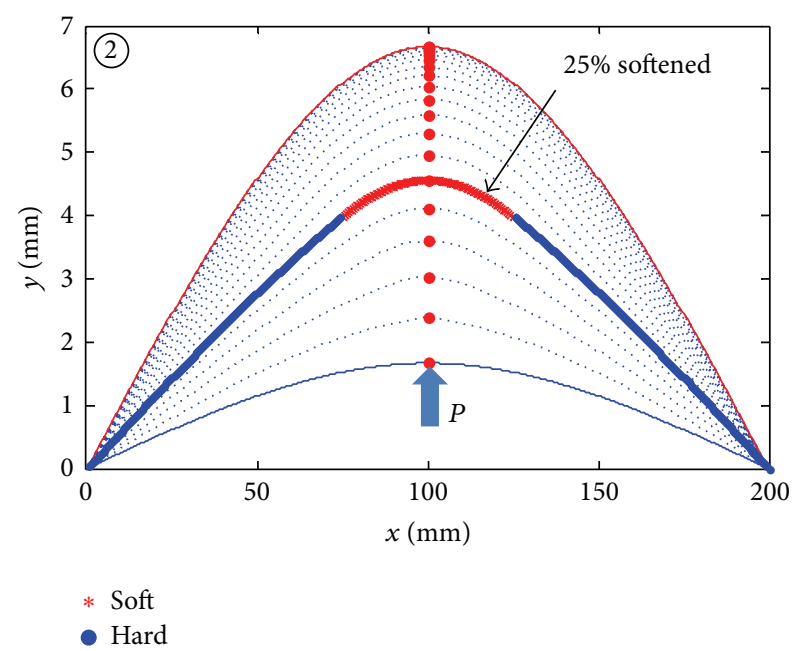

FIGURE 6: Morphed shapes with highlighted peak points (fixed middle soft segment grows symmetrically to both sides in 5\% increments).

with two different lengths have been shown. Case 1 presents a pattern in which the section is constrained on the left (or right) support while the length of the section grows. In Case 2 , the soft segment grows symmetrically from the midspan. In Case 3, a soft segment with a fixed length shifts through the structure (from left end to right end or vice versa) over consecutive stages.

Figure 5 shows the results of morphed shapes for Case 1 in which the soft segment grows from $0 \%$ to $100 \%$ of the beam length in increments of 5\%. An example of morphed shape, with soft segment highlighted, is shown for the $50 \%$ soft trial. Two distinct features appear in the results. First, the amplitude of shape change increases with the increased 


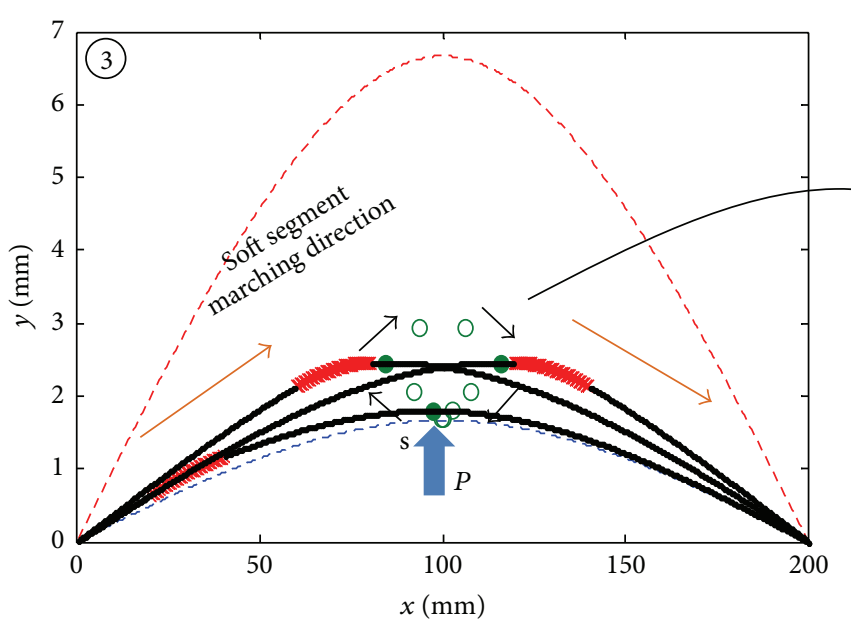

(a)

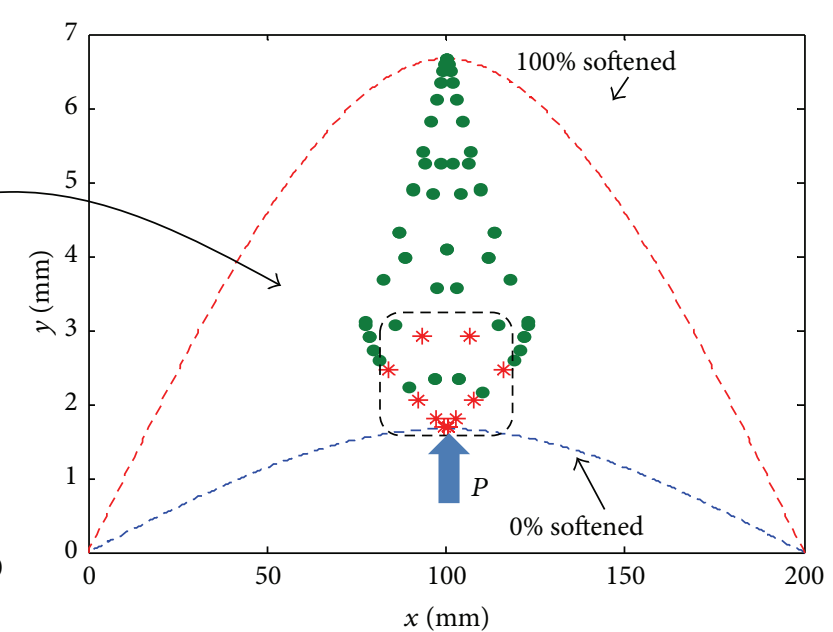

(b)

Figure 7: (a) Peaks locus in an asymmetric unisection scheme with constant 10\% segment length marching from left to the right. Sample morphed shapes are shown. Start point, S, and moving direction of the peaks are specified. (b) Peaks distribution for different soft segment's length (0-100\%). Peak locus corresponding to $10 \%$ length is highlighted within dashed box.

TABLE 1: Specifications of the beam model.

\begin{tabular}{lccc}
\hline Variable & Description & Value & Unit \\
\hline \multicolumn{4}{c}{ Geometrical properties } \\
$w$ & Beam length & 200 & $\mathrm{~mm}$ \\
$h$ & Width of rectangular section & 10 & $\mathrm{~mm}$ \\
\hline \multicolumn{4}{c}{ Height of rectangular section } \\
$E_{h}$ & Material properties & $\mathrm{mm}$ \\
$E_{s}$ & Hard phase modulus & 3000 & $\mathrm{MPa}$ \\
$v$ & Soft phase modulus & 750 & $\mathrm{MPa}$ \\
$P$ & Poisson's ratio & 0.3 & - \\
\hline
\end{tabular}

softened region, as expected. Second, for all shapes except for the fully hard or fully soft beam, the shape is skewed to the left (nonsymmetric about the midspan). Clearly the modulus change enables an expanded space of possible shapes, not only in amplitude but in quality (in this case symmetry) too.

In order to track and quantify the spatial configuration of the beam in different morphing scenarios, it is reasonable to define a unique metric that characterizes the shape of the deflected beam. A peak locus is referred to as the set of the peak points during a particular morphing process (from $0 \%$ to $100 \%$ softened). A plot of peak locus demonstrates the spatial distribution of these highlighted points (see Figure 5). Notice the peak loci in pattern 1 form the borders of an envelope. It is worth noting that the mirror image of the peak loci can be formed by initiating the scheme of Case 1 from the right end of the beam.

Another way to interpret the curves is the space of possible beam shapes that are enabled by morphing without changing the applied force. In this work, this space is referred to as morph space. The lowest beam shape shown (blue dashed line at the bottom) is the shape generated when the force is applied to the original hard beam. All of the other shapes, with larger and some nonsymmetric deformations, are available by changing modulus of various sections according to the scheme of Case 1.

Figure 6 pictures the morphed shapes with their highlighted peak points for Case 2. Once again, the results are shown for increasing soft segment lengths from $0 \%$ (bottom curve) to $100 \%$ (top curve) in $5 \%$ increments, but this time the soft segments are centered at the beam's midspan. As an example, one of the intermediate curves (25\% softened) has been indicated. Unlike the previous pattern, all the deformed shapes are symmetric with respect to the midspan and no deviation of the peak points is observed. One could anticipate this by considering the central and symmetric orientation of the soft segments.

Morphing under Case 3 results in asymmetric shapes. Recall that, in this case, a constant length soft segment is marched along the beam from left to right. To understand the behavior, we present the results in terms of fixed-length soft segments marched along the beam, where the section length increases for each subset of data. For example, assume a constant segment length of $10 \%$ (of the total beam length). If one simulates the deflection of the beam for each marching step, Figure 7(a) is obtained. Figure 7(a) contains a group of morphed shapes as the $10 \%$ soft segment marches from left to right along the beam. The larger (red) points highlight the peak beam deflections, indicating asymmetric morphing for cases in which the soft segment is off-centered (e.g., three cases are shown with the soft segments highlighted with (red) cross symbols, at $0.20,0.40$, and $0.70 \times L$ from the left end of the beam). The arrows indicate the direction of peak movement as the $10 \%$ soft segment marches from left to right, starting with point $S$.

Now if one simulates the deflection of the beam with soft segments of different lengths (starting at 5\% of the total beam length and increasing in section length to 50\%) and 


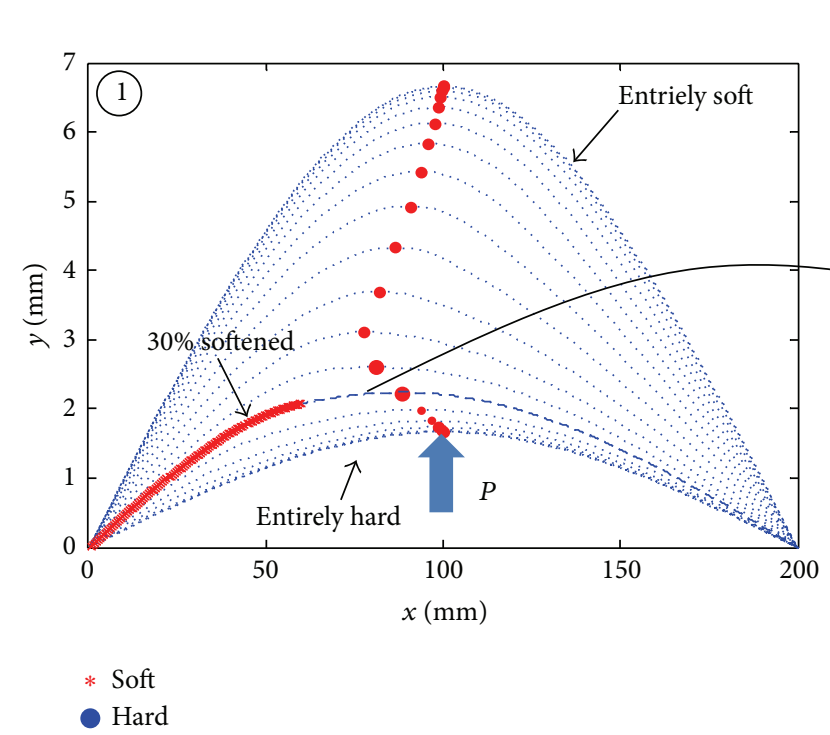

(a)

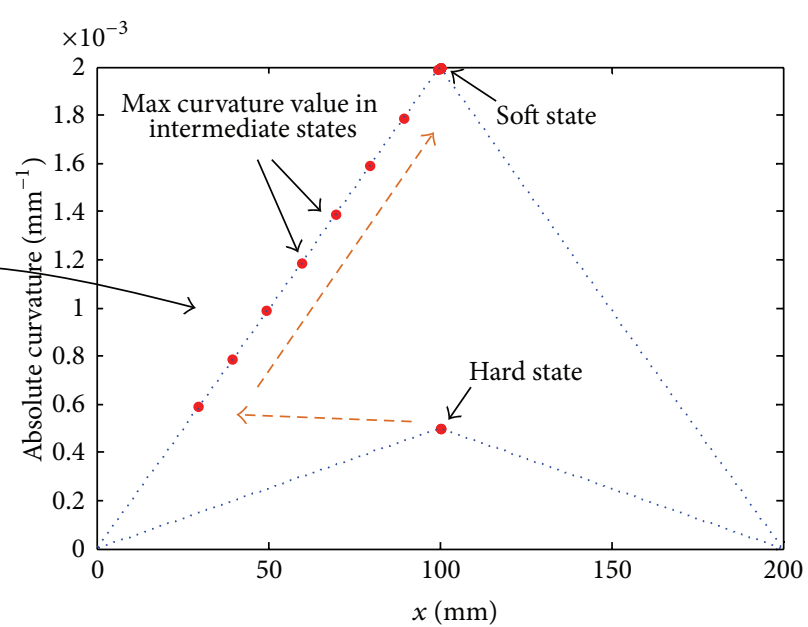

(b)

FIGURE 8: (a) A family of morphed shapes in pattern 1; (b) maximum curvature values in different morph states are all lower than that of $100 \%$ soft state case.

marching from left or right, and the peak loci are plotted at each iteration, Figure 7(b) is obtained. As the segments become wider, the peak loci spread out in both vertical and side directions. It is interesting to note that the peak points of Case 3 occupy the inside of the envelope formed by the peak points of Case 1. Note that, for all the soft segment lengths, the peaks never surpass the envelope borders.

There are few points worth considering with regard to Case 3. Firstly, in all cases of unisection pattern, the boundaries of the "morphing space" are represented by $0 \%$ and $100 \%$ soft segment length. Moreover, the accessible peak points (or equivalently shapes) with this pattern are significantly spatially diverse. While the morph Cases 1 and 2 created shapes along the side borders and middle axis of the envelope (previously shown in Figure 5), the third model could produce shapes whose peaks lie not only along the borders but also within the envelope. In addition, the distribution pattern provides a measure to quantitatively adjust the required section length to achieve the desired horizontal and vertical positions of the peak.

Stability Considerations. According to linear elasticity theory, stress field in the deformed beam is proportional to the curvature distribution [19]. In a morphing process where diverse shapes with different curvatures are obtained, it is essential to ensure that all the intermediate shapes are elastically stable. Figure 8(b) displays the maximum curvature distribution of the shapes for Case 1 scheme with softening progression of 0 to $100 \%$ (dashed arrows). Note that the maximum curvature magnitude of the intermediate curves is less than the one in the soft state, which, due to direct relationships of curvature and stress, implies the maximum stress is lower than the one in the soft state and that all the morphed shapes are elastically stable.

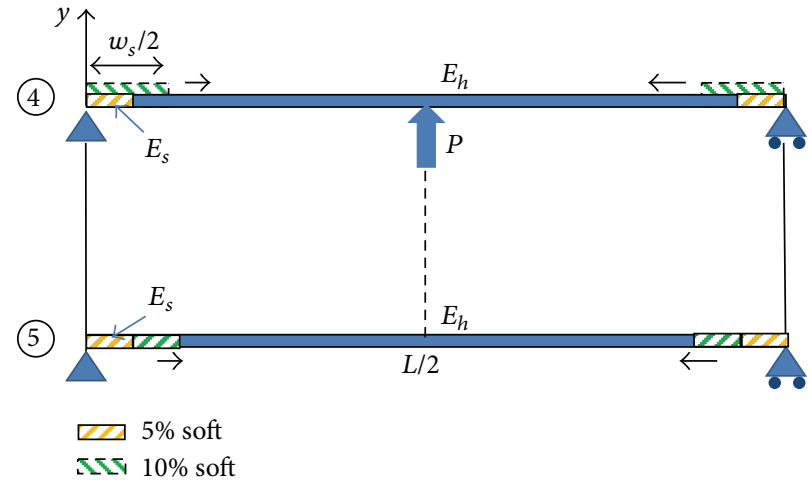

FIgURE 9: Symmetrical bisection morph patterns.

The maximum curvature distributions of the morphed shapes generated from all other unisection morph patterns were also studied. It was concluded from the curvature values that their stresses are within the secure region.

(b) Bisection Morphing (Symmetrical and Unsymmetrical). A schematic of a symmetrical bisection morph pattern is shown in Figure 9 in which two separate sections are transitioned simultaneously and their sizes grow as different trials are evaluated. The first case is similar to the corresponding case in unisection morph where the sections remain tied at the supports and their widths grow in consecutive steps. Likewise, the second case is a marching section pattern where fixed-width sections continue to move along the structural domain.

The morphed shapes with their highlighted peaks are simulated in Figure 10. As indicated, this morphing strategy results in symmetric shapes, that is, no spatial variation in 

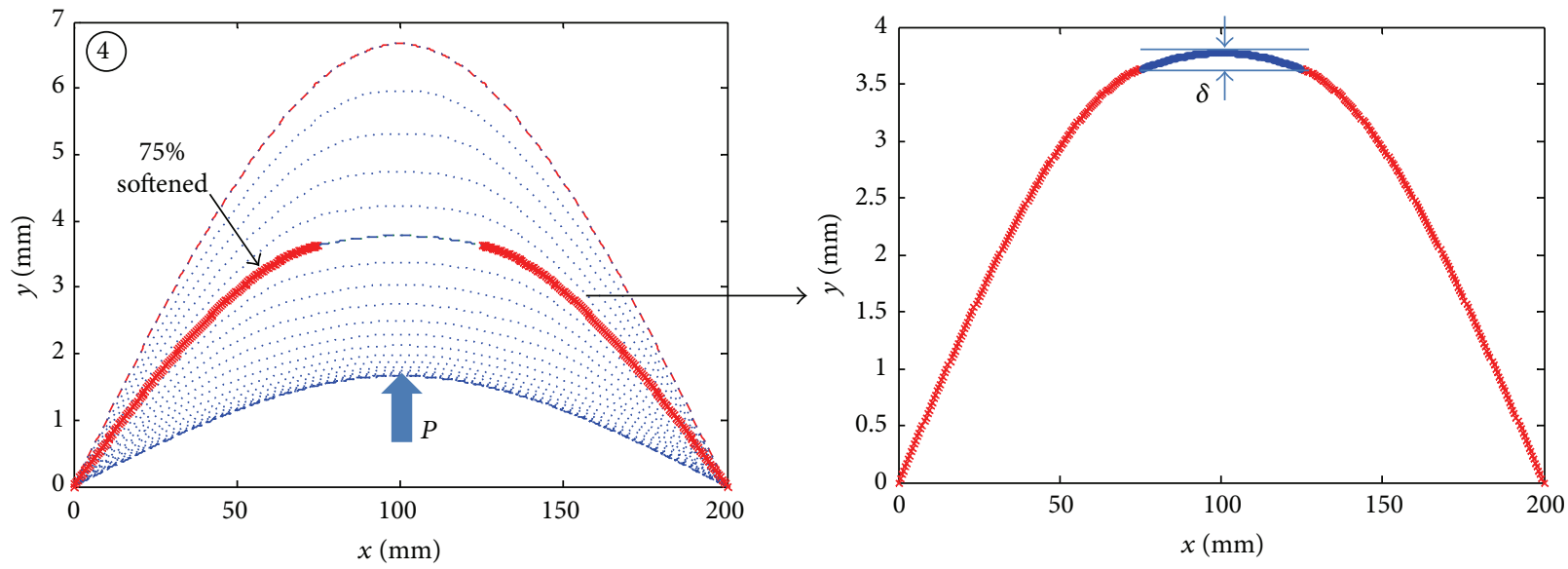

FIGURE 10: Morphed shapes in a bisection pattern (two soft segments are tied to the supports and grow in length in consecutive 5\% steps).

the peak locus, but with widened curves in the vicinity of the peaks. The left plot highlights a trial in which $75 \%$ of the beam is softened, and this trial is expanded in the right plot.

In order to quantify the width of the shapes, a flat zone is defined as a part of the curve, in the neighborhood of the peak, where the displacement fluctuation remains within a certain assumed tolerance $(\delta=0.15 \%)$ :

$$
\left|\frac{y_{i+1}-y_{i}}{y_{i}}\right| \leq \delta
$$

Each flat zone is identified by its start and end points. The loci of these end points are plotted in Figure 11.

The patterns presented in Figure 12 are another bisection morphing strategy where the morphing sections are unsymmetrical with respect to the midplane of the structure. This scheme includes two major patterns: in the first pattern, soft segments keep their starting locations and propagate through the beam, whereas in the second case constant length sections march from left to right (or right to left) in sequential steps.

Recall that Figure 13(a) showed the produced shapes as fixed soft segment at the left grew to the right with certain size increments. Similarly, resulting shapes from morphing pattern 6 are shown in Figure 13(b). Starting from hard state, two soft segments, one fixed at left end and the other in the center, grew from left to right with 5\% incremental increase and peak locus of morphed shapes was traced by solid dots. Firstly, similar to its unisection counterpart (Figure 13(a)), the diversion of peak loci is a significant feature in this pattern. Secondly, unlike the first pattern in the unisection scheme where the peaks were situated on the left side of the actuation force, the loci fall on the right. The central soft segment seems to be dominating in steering the peak locus. Notice that a mirror pattern would have resulted if the marching went from right to left.

Similarly, maximum curvature distribution in the shapes was obtained when the bisection morph strategy was investigated. The corresponding values to the intermediate shapes were all lower than that of completely soft state case, which confirms they remain stable.

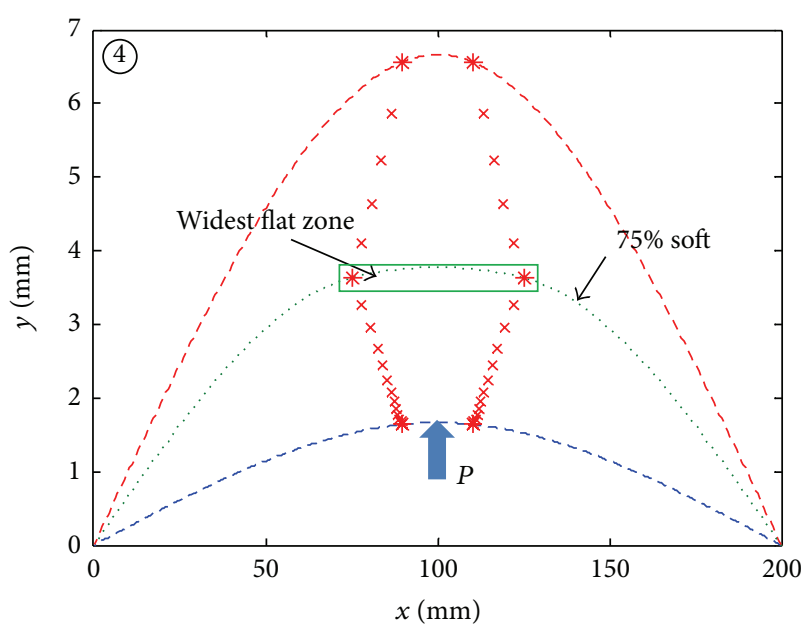

Figure 11: Loci of the boundaries of the flat zones.

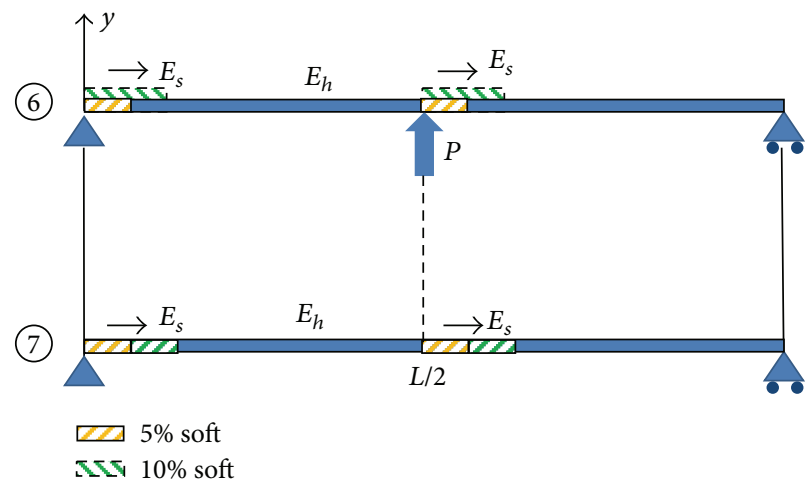

Figure 12: Asymmetrical bisection morph patterns (change to 6,7 ).

Two major morphing patterns (i.e., unisection and bisection) have been so far considered. For each pattern, the distribution of the peaks of the morphed shapes was explored and the achievable shape space was determined. Figure 14 illustrates the aggregation of results, showing peak distribution 

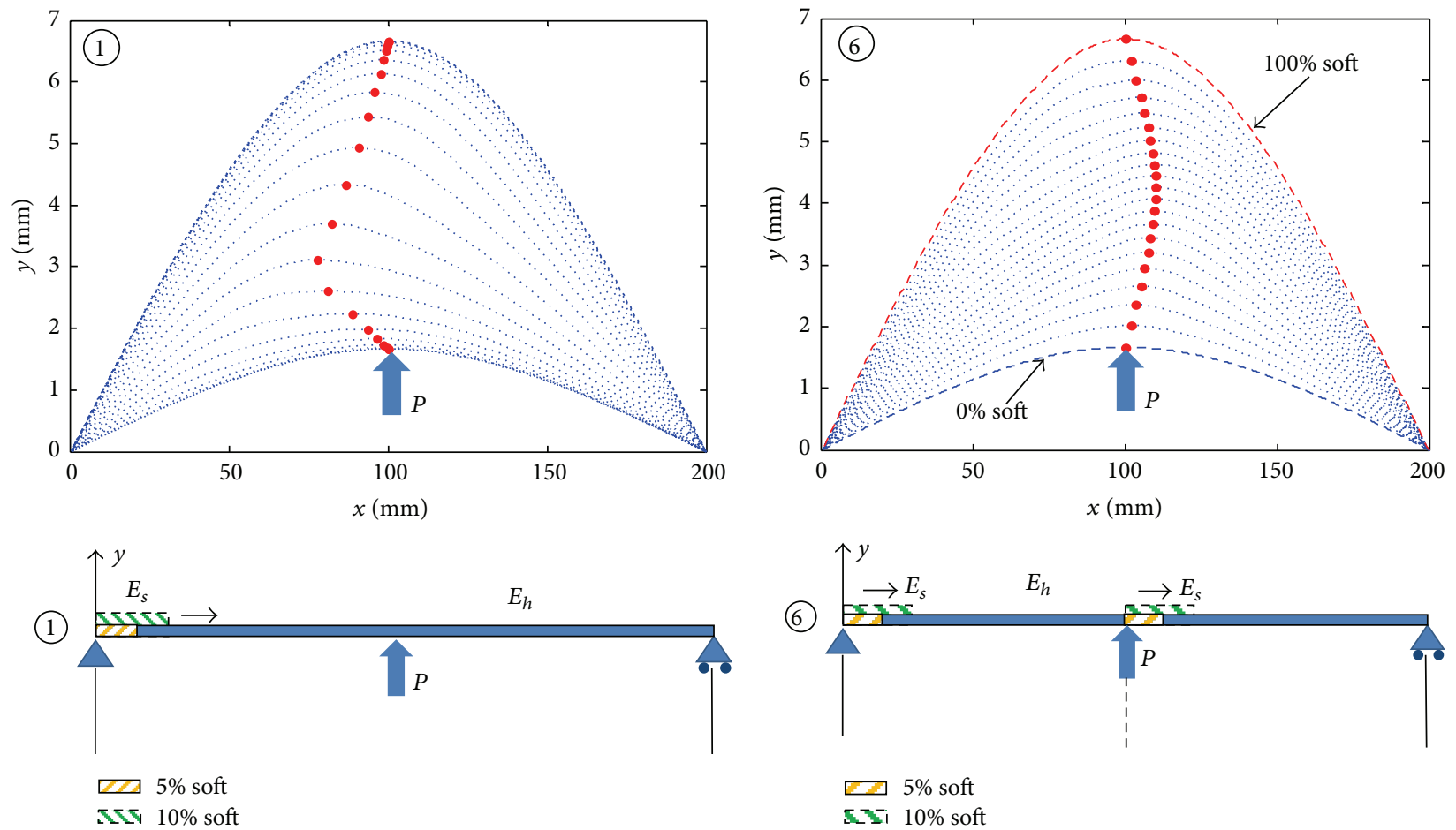

(a)

(b)

FIGURE 13: Morphed shapes of the beam with highlighted peak points: (a) soft-section pattern; (b) asymmetric bisection pattern (two soft segments, one fixed at left end and the other in the center, grow sequentially from left to right).

with highlighted potential achievable spaces by morphing with different patterns. One could observe that the unisection morph scheme yields a wider accessible space. Adding the second morphing section would cause an additional degree of flexibility in the opposing direction as the first section which reduces the overall deviation in the morphed structure.

Secondly, the unisection morph pattern covers broader points in its accessible space. The bisection scheme can produce shapes with more scattered peaks in lower soft segment lengths, whereas for the larger segment lengths the locus concentrates on a narrower defined boundary. This could also be related to the middle section which limits the dominating role of the side section in deviating the peaks. The other observation one would notice is on the peak value of the most deviated morphed shape in each morphing scheme. While the unisection pattern can create well-deviated or top-flat shapes, the bisection model can produce tall and slim shapes under the same morph parameters. This is due to reduced bending rigidity of the structure by adding transitioned section.

Boundary Edge. As noticed in Figures 14 and 5, the extent of the deviated shapes is prescribed by the first pattern of the unisection morphing scheme. For this pattern, there is a particular segment length that results in the most asymmetric shape whose peak defines the edge of the morph boundary. The significance of this length is investigated in this section.
The closed-form solution for Case 1 could be summarized as

$$
\left[\begin{array}{cccc}
E_{s}^{-1} & -E_{h}^{-1} & 0 & 0 \\
x_{2} E_{s}^{-1} & -x_{2} E_{h}^{-1} & E_{s}^{-1} & -E_{h}^{-1} \\
0 & 0 & 1 & 0 \\
0 & L & 0 & 1
\end{array}\right]\left[\begin{array}{l}
c_{1}^{1} \\
c_{1}^{2} \\
c_{2}^{1} \\
c_{2}^{2}
\end{array}\right]=\left\{\begin{array}{c}
A f\left(x_{2}\right) \\
A g\left(x_{2}\right) \\
0 \\
-g(L)
\end{array}\right\} .
$$

Solving for the coefficients $c_{1}^{1}$ and $c_{1}^{2}$ will result in

$$
\begin{aligned}
c_{1}^{1} & =\frac{A\left[g\left(x_{2}\right)-x_{2} f\left(x_{2}\right)+L f\left(x_{2}\right)\right]-E_{h}^{-1} g(L)}{L E_{s}^{-1}} \\
c_{1}^{2} & =\frac{A\left[g\left(x_{2}\right)-x_{2} f\left(x_{2}\right)\right]-E_{h}^{-1} g(L)}{L E_{h}^{-1}},
\end{aligned}
$$

where functions $f\left(x_{2}\right)$ and $g\left(x_{2}\right)$ are defined as

$$
\begin{aligned}
& f(x)=-\frac{P b}{2 L} x^{2} \\
& g(x)=-\frac{P b}{6 L} x^{3} .
\end{aligned}
$$

Recall $L$ is the total length and $b$ is the force distance from the right support. As described, the shape of the beam is described by two piecewise functions; that is,

$$
y(x)=H_{1} y_{1}(x)+H_{2} y_{2}(x),
$$




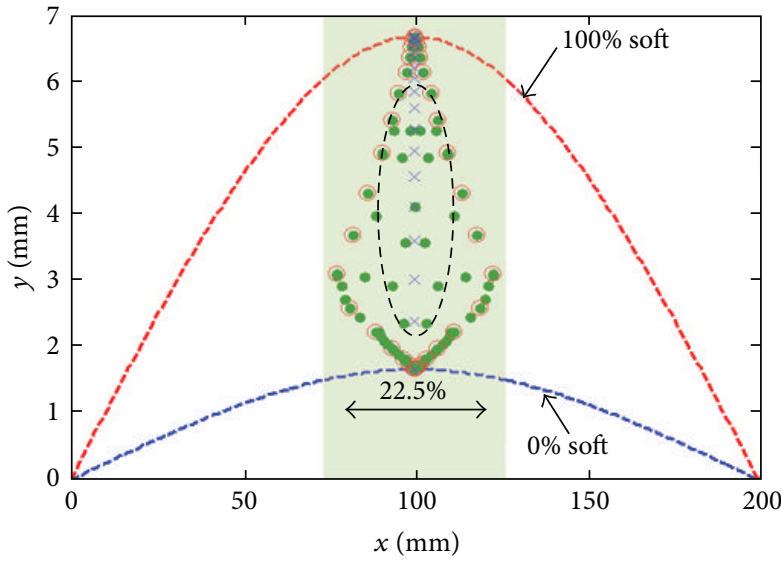

C Case 1

$\times$ Case 2

- Case 3
(1)

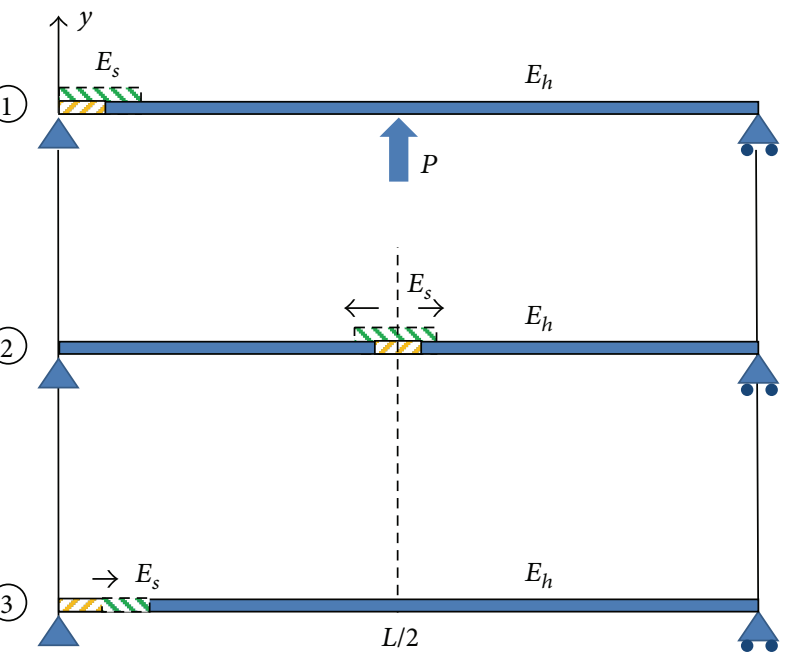

$\square$ 5\% soft

xIV $10 \%$ soft

(a)
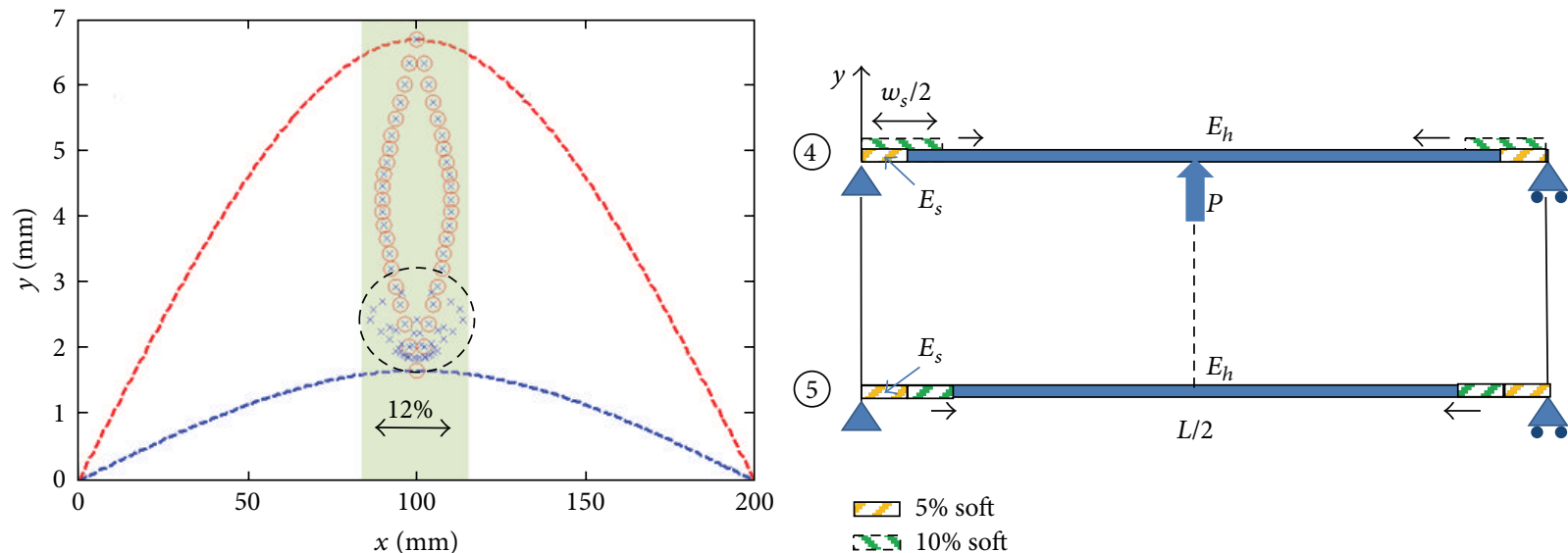

Case 4

$\times$ Case 5

(b)

FIGURE 14: Morphing schemes comparison: unisection morph (a) and bisection morph (b).

where

$$
\begin{aligned}
& y_{1}(x)=\frac{1}{E_{s} I}\left[g(x)+c_{1}^{1} x\right] \\
& y_{2}(x)=\frac{1}{E_{h} I}\left[g(x)+c_{1}^{2} x+c_{2}^{2}\right] .
\end{aligned}
$$

Let $x_{2}$ be the length-normalized coordinate measured from the left support and let $x_{2}^{p}$ be the location of the peak. Solving for $x_{2}^{p}$ from each of the two shape functions $y_{1}$ and $y_{2}$ results in

$$
\begin{aligned}
& x_{2}^{p}=\frac{2 L}{P b} c_{1}^{1} \\
& x_{2}^{p}=\frac{2 L}{P b} c_{1}^{2} .
\end{aligned}
$$

For this model with specification introduced in Table 1, if one plots the variation of $x_{2}^{p}$ in terms of the coefficients $c_{1}^{1}$ and $c_{1}^{2}$ defined in (9), Figure 14 is obtained. Compatibility of the peak location is only satisfied in the intersection of the plots where the segment length is $37.5 \%$ of the beam length. This can be verified in Figure 5 by determining the segment length corresponding to the most asymmetric shape. Assuming small deformations, the value of $x_{2}^{p}$ also represents the extent of the achievable space from the midplane.

Effect of Model Parameters on Boundary Edge. According to (9), location of the boundary edge is a function of the beam geometry, actuation, and the two (upper and lower) moduli. Figure 16 shows the coefficient curves in the vicinity of their 


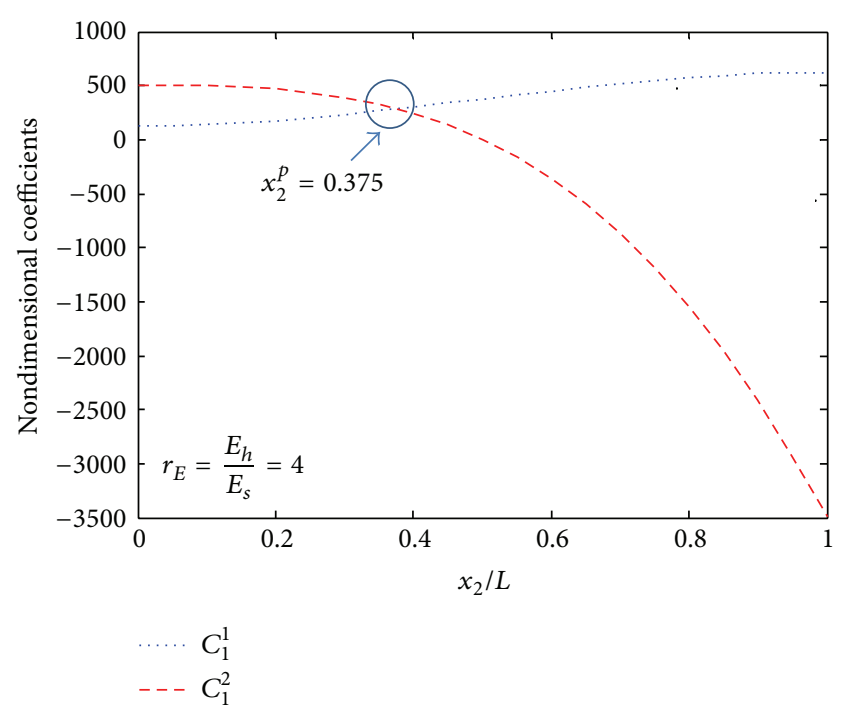

FIGURE 15: Variation of the shape coefficients versus longitudinal coordinate.

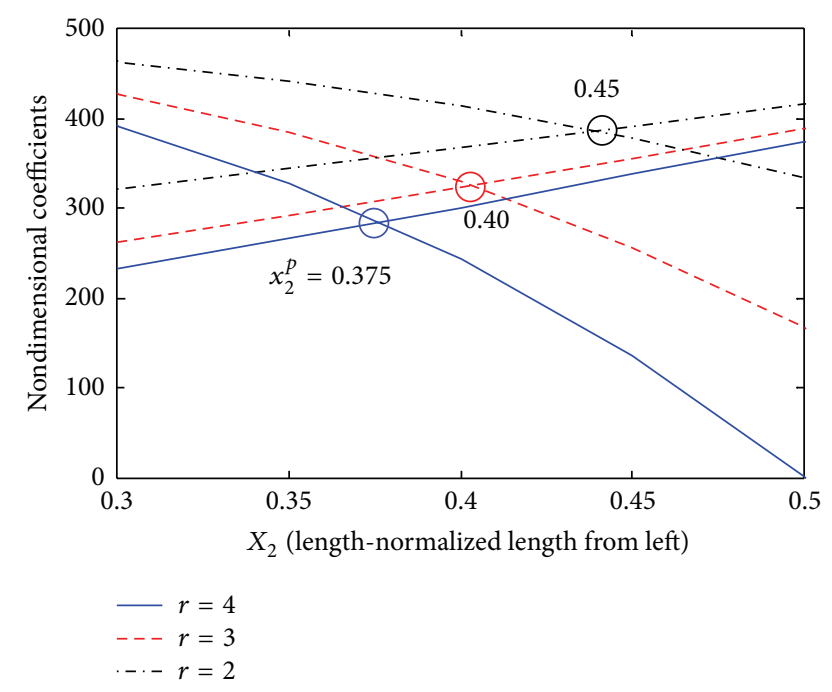

FIGURE 16: Effect of hard-to-soft moduli ratio $\left(r=E_{h} / E_{s}\right)$ on the boundary extent (intersection point).

intersection (similar to those in Figure 15) for different hardto-soft moduli ratio $\left(r=E_{h} / E_{s}\right)$. For the same set of actuation forces and beam geometries, the boundary edge expands to the sides as the lower modulus decreases. Another significant observation is regarding the vertical beam displacement: notice (in the figure) that the value of nondimensional coefficients $\left(c_{1}^{1}\right.$ and $\left.c_{1}^{2}\right)$ is constantly increasing with moduli ratio. These coefficients were directly used in (2) to calculate the vertical beam displacement. This means that one can expand the morph space horizontal and vertical bounds by selecting larger hard-to-soft moduli ratio.

Figure 17 displays the effect of actuation force. Unlike modulus, increasing the force does not change the extent of the morph space in lateral (side) direction; however, it increases the vertical boundaries of the achievable shapes.

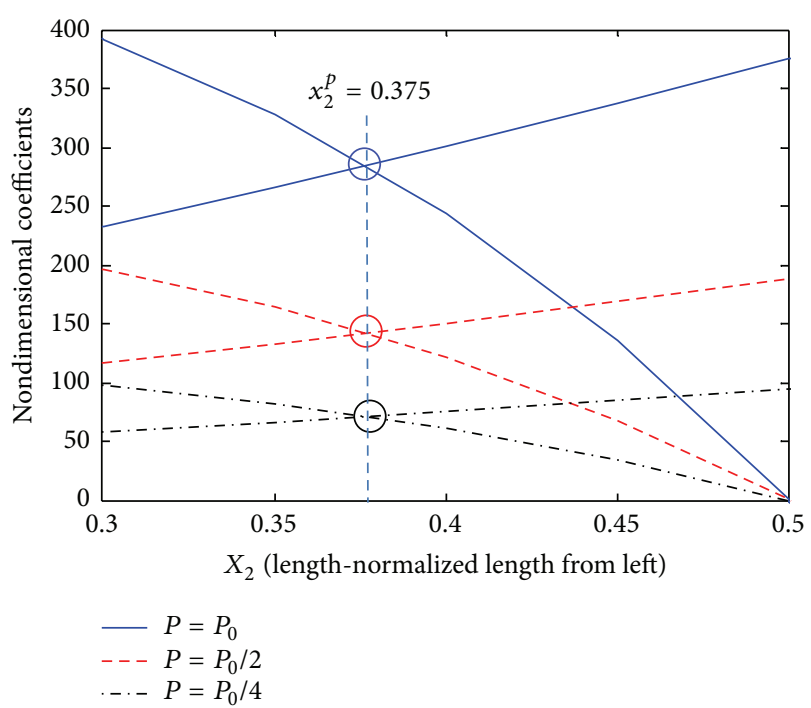

FIGURE 17: Effect of actuation force on the boundary edge.

This can also be explained by observing the coefficients trend and realizing their direct contribution in the vertical deformation.

Figure 18 presents variation of side boundary for different beam lengths. The margins of the morphed shapes do not vary and for the similar load and moduli remain $37.5 \%$ of the length. Moreover, one may expect to see higher vertical displacements in a larger-span beam.

Figure 19 shows the effect of force location on the side boundary limit. Recall that $b$ is the distance of the actuator to the right support. As the force moves towards the right, the morph space shifts in the same direction. By observing the coefficients plot, one could verify that, as the force moves off the centerline, the vertical extent of the morphed shapes would reduce.

\section{Conclusion}

This work explored the shape morphing of a variable-modulus simply supported elastic beam from a forward approach perspective. Specifically, the spatial distribution of the structure due to local/total modulus was studied. It was shown that, for a given fixed force in the midspan, modulus manipulation can create shapes that (1) have larger deflected magnitude due to increased beam compliance and (2) can be more irregular, for example, asymmetric, because of expanded morph space. The resulting morphed shapes were quantified in terms of the characteristic parameters, such as spatial distribution of the apex of the shapes, deviation from the axis of the symmetry, and, in case of flat-top shapes, the width of the flat portion. Depending on the geometrical characteristic of the target shape, one could choose the appropriate morphing strategy. An analysis explained the relationship between the maximum extent of the peak locus and soft segment length. Desired boundary of the morph space can be achieved by tuning the hard-to-soft moduli ratio and force location. In addition, changing force magnitude 


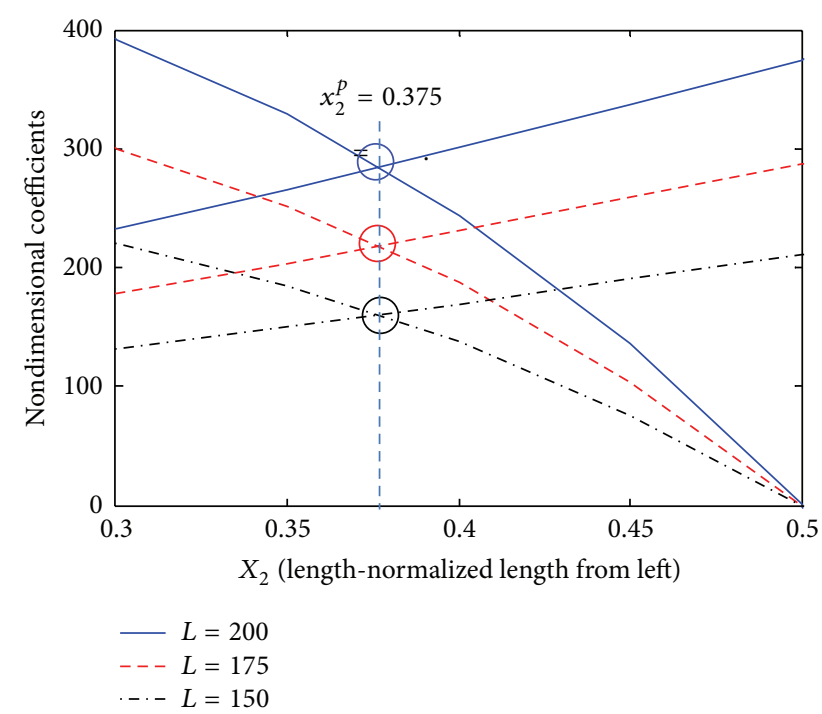

FIGURE 18: Effect of beam length on the boundary edge.

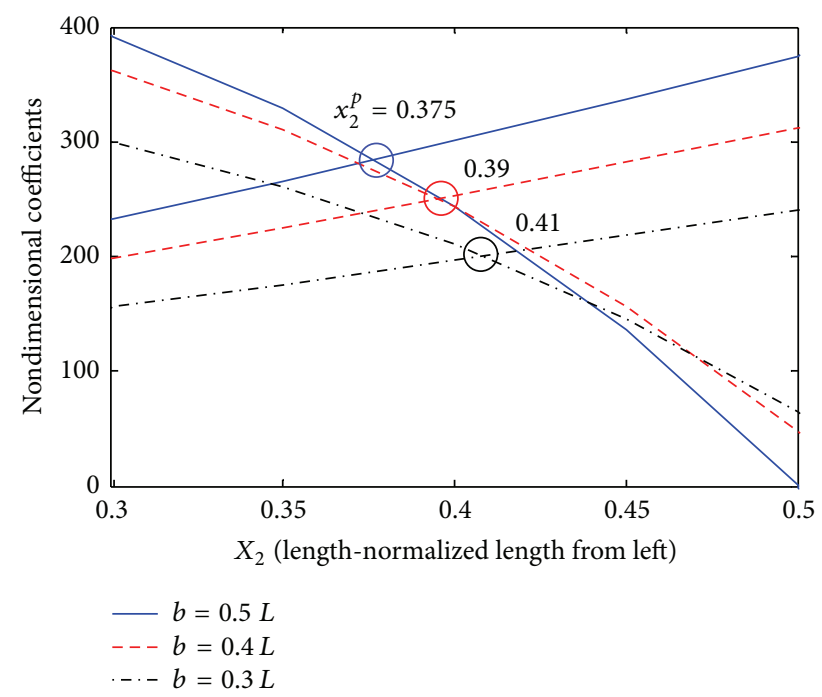

FIGURE 19: Effect of force location on the side boundary edge.

and beam length can also move the morphed shapes' limit vertically. Future work will investigate structure's morphing with multiple loading and soft modulus values.

\section{Conflict of Interests}

The authors declare that there is no conflict of interests regarding the publication of this paper.

\section{References}

[1] J. L. Reed, C. D. Hemmelgarn, B. M. Pelley et al., "Adaptive wing structures," in 8th International Society of Photo-Optical Instrumentation Engineers, vol. 5762 of Proceeding of SPIE, p. 322, Bellingham, Wash, USA, 2005.
[2] J. Blondeau, J. Richeson, and D. J. Pines, “Design, development and testing of a morphing aspect ratio wing using an inflatable telescopic spar," in Proceedings of the 44th AIAA/ASME/ ASCE/AHS/ASC Structures, Structural Dynamics, and Materials Conference, Norfolk, Va, USA, April 2003.

[3] J. D. Jacob, A. Simpson, and S. Smith, "Design and flight testing of inflatable wings with wing warping," Tech. Rep., Society of Automotive Engineers, 2005.

[4] N. Ameri, E. Livne, M. H. Lowenberg, and M. I. Friswell, "Modelling continuously morphing aircraft for flight control," in Proceedings of the AIAA Guidance, Navigation and Control Conference and Exhibit, pp. 1-23, August 2008.

[5] B. N. Agrawal and K. E. Treanor, "Shape control of a beam using piezoelectric actuators," Smart Materials and Structures, vol. 8, no. 6, pp. 729-740, 1999.

[6] F. T. Calkins, J. H. Mabe, and G. W. Butler, "Boeing's variable geometry chevron: morphing aerospace structures for jet noise reduction," in Smart Structures and Materials: Industrial and Commercial Applications of Smart Structures Technologies, vol. 6171 of Proceedings of SPIE, San Diego, Calif, USA, March 2006.

[7] J. H. Mabe, F. T. Calkins, and R. T. Ruggeri, "Full-scale flight tests of aircraft morphing structures using SMA actuators," in Proceedings of the SPIE, Active and Passive Smart Structures and Integrated Systems, vol. 6525, ETATS-UNIS: Society of PhotoOptical Instrumentation Engineers, Bellingham, Wash, USA, 2007.

[8] Z. Chaudhry and C. A. Rogers, "Bending and shape control of beams using SMA actuators," Journal of Intelligent Material Systems and Structures, vol. 2, no. 4, pp. 581-602, 1991.

[9] S. Wang and J. C. Brigham, "A computational framework for the optimal design of morphing processes in locally activated smart material structures," Smart Materials and Structures, vol. 21, no. 10, Article ID 105016, 2012.

[10] M. M. Keihl, R. S. Bortolin, B. Sanders, S. Joshi, and Z. Tidwell, "Mechanical properties of shape memory polymers for morphing aircraft applications," in Proceedings of the Industrial and Commercial Applications of Smart Structures Technologies, pp. 143-151, San Diego, Calif, USA, March 2005.

[11] D. Perkins, J. Reed Jr., and E. Havens, "Morphing wing structures for loitering air vehicles," in Proceedings of the 45th AIAA/ASME/ASCE/AHS/ASC Structures, Structural Dynamics \& Materials Conference, Palm Springs, Calif, USA, April 2004.

[12] S. G. Rauscher, "Testing and analysis of shape-memory polymers for morphing aircraft skin application," in Mechanical Engineering, University of Pittsburgh, Pittsburgh, PA, USA, 2008.

[13] B. M. Boyerinas, W. W. Clark, L. M. Weiland, and S. Joshi, "Design and fabrication of a variable stiffness link for use in an unmanned air vehicle," in Proceedings of the ASME Conference on Smart Materials, Adaptive Structures and Intelligent Systems, pp. 317-323, 2009.

[14] Y. Chen, J. Sun, Y. Liu, and J. Leng, "Variable stiffness material and structural concepts for morphing applications," Smart Materials and Structures, vol. 21, no. 9, 2012.

[15] J. Sun, X. Gong, Y. Liu, and J. Leng, "Variable camber wing based on shape memory polymer skin," in Proceedings of the 54th AIAA/ASME/ASCE/AHS/ASC Structures, Structural Dynamics, and Materials Conference, Boston, Mass, USA, April 2013.

[16] J. Leng, X. Lan, Y. Liu, and S. Du, "Shape-memory polymers and their composites: Stimulus methods and applications," Progress in Materials Science, vol. 56, no. 7, pp. 1077-1135, 2011. 
[17] P. Calvo-Marzal, M. P. Delaney, J. T. Auletta et al., "Manipulating mechanical properties with electricity: electroplastic elastomer hydrogels," ACS Macro Letters, vol. 1, no. 1, pp. 204-208, 2012.

[18] R. D. Harris, J. T. Auletta, S. A. M. Motlagh et al., "Chemical and electrochemical manipulation of mechanical properties in stimuli-responsive copper-cross-linked hydrogels," ACS Macro Letters, vol. 2, no. 12, pp. 1095-1099, 2013.

[19] S. P. Timoshenko, Theory of Elastic Stability, McGraw-Hill, New York, NY, USA, 1961. 

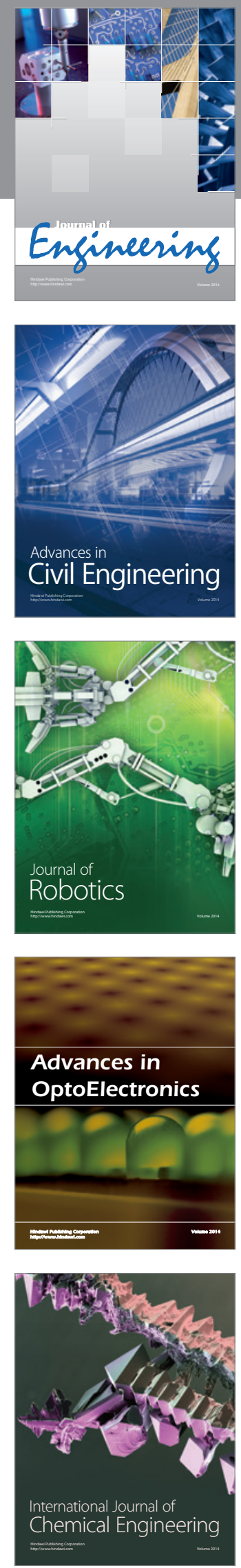

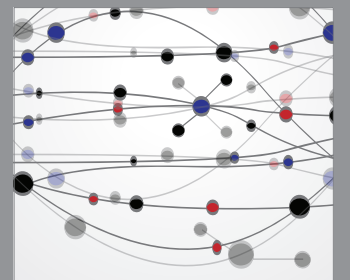

The Scientific World Journal
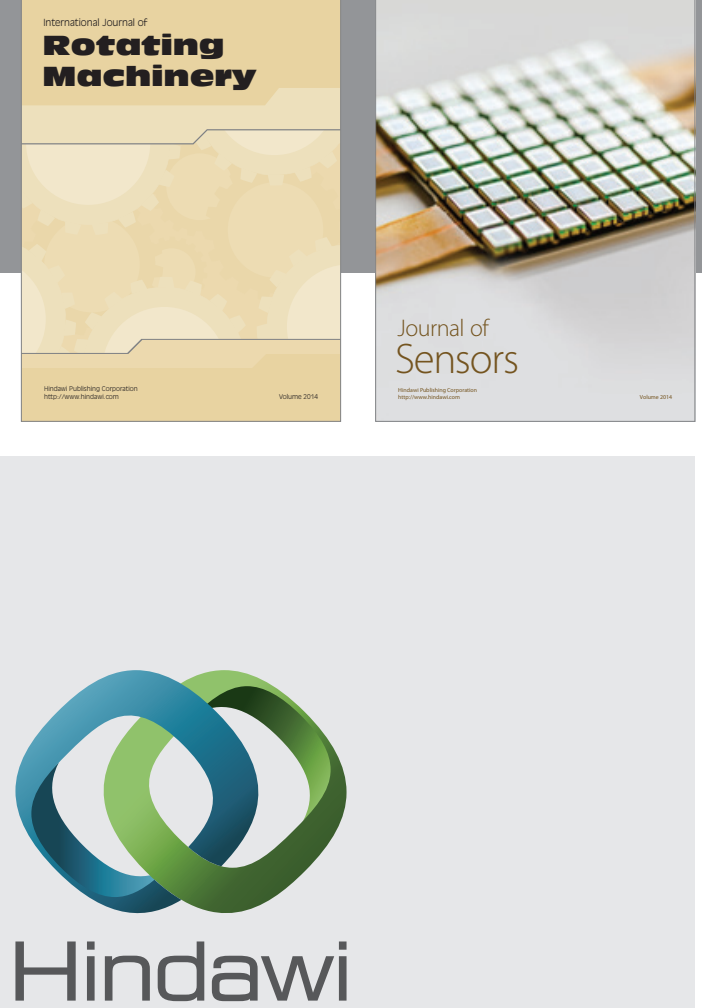

Submit your manuscripts at http://www.hindawi.com
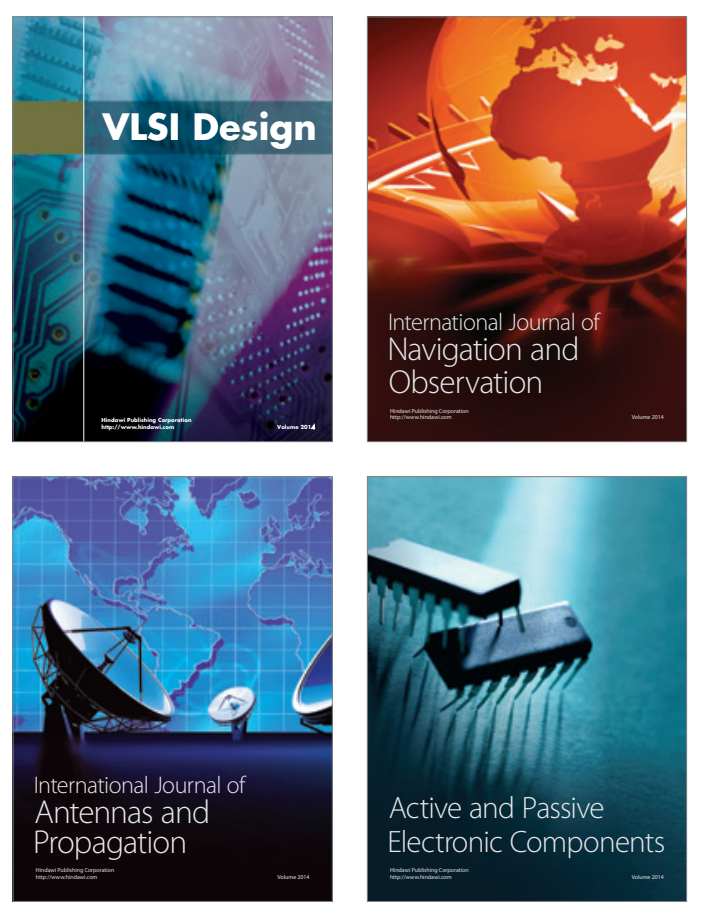
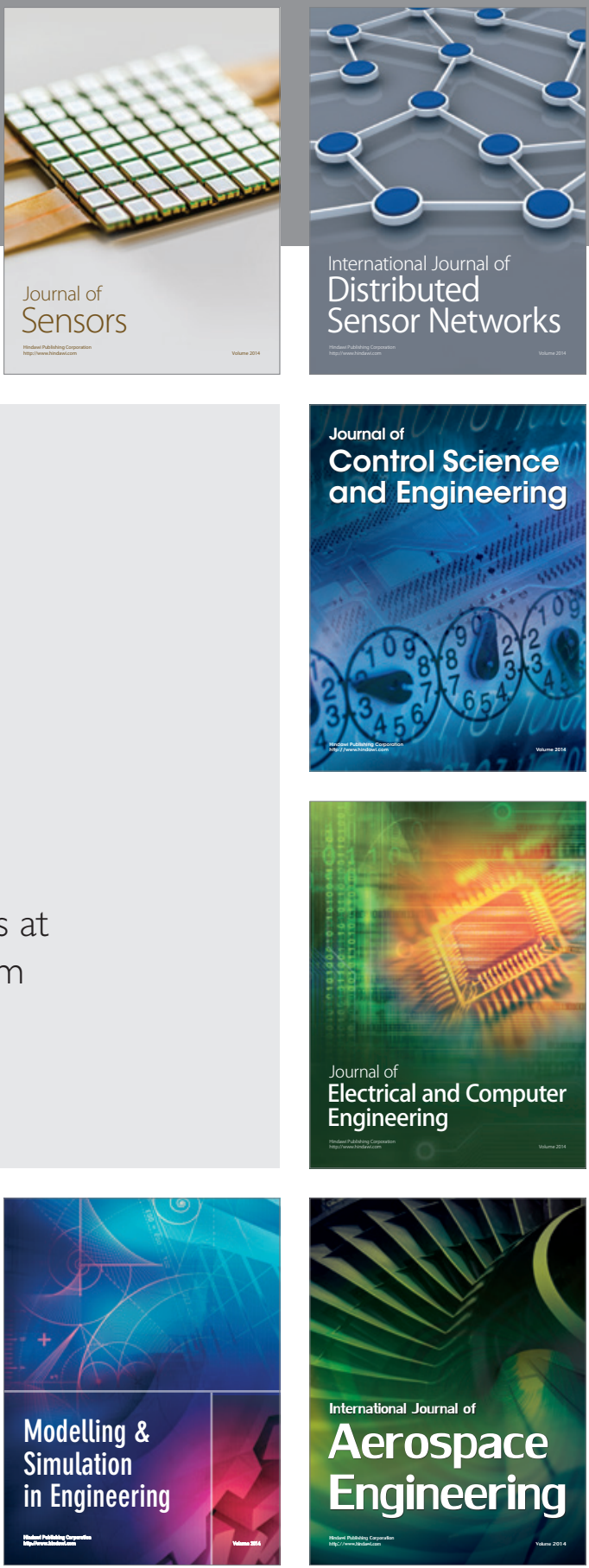

Journal of

Control Science

and Engineering
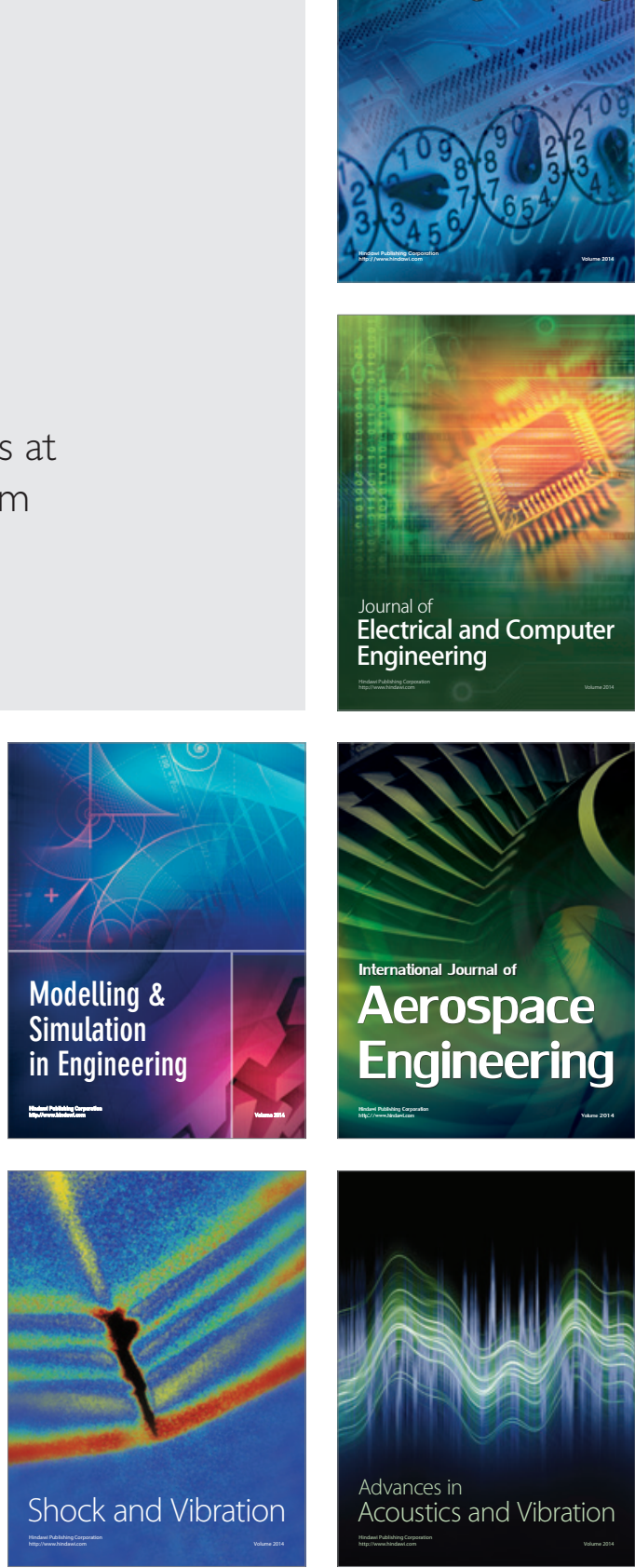